\title{
Analysis and Quantification of Transition Metal Borides with WDS and EDS
}

\author{
Ralf Terborg $^{1^{*}}$ and Silvia Richter ${ }^{2}$
}

1. Bruker Nano GmbH, Am Studio 2D, Berlin, Germany.

2. Central Facility for Electron Microscopy, RWTH Aachen University, Aachen, Germany.

* Corresponding author: ralf.terborg@bruker.com

Quantitative X-ray microanalysis using wavelength-dispersive (WDS) or energy-dispersive (EDS) spectrometers of light elements or using lines in the low energy range $(<1 \mathrm{keV})$ is a challenge due to low X-ray yields and overlapping lines, especially for EDS. Furthermore, intensities can be affected by considerable absorption effects [1,2,3] and uncertainties in mass absorption coefficients (MACs).

In former work WDS and EDS analysis of steel was compared at an accelerating voltage of $6 \mathrm{kV}$ and $\mathrm{L}$ lines of the $3 \mathrm{~d}$ transition elements were used for quantification. Since EDS spectra present a significant overlap of L lines, spectral deconvolution was necessary [3,4] to get reasonable results. In addition, SDDEDS quantification of chromium and lanthanum borides have been performed in [5].

In this work spectrum acquisition and quantitative analysis of different transition metal borides have been performed using EDS and WDS. Besides the challenges already mentioned peak shifts of a few eV and different peak shapes have to be considered as well. For WDS, integral measurements rather than peakto-background measurements of the boron peak are recommended [1]. For EDS, peak shape variation is less important due to the lower energy resolution, Figure 1. However, the modelling of a correct bremsstrahlung background is an unavoidable issue for an accurate analysis. For our study the physical background model based on [6] was used.

WDS and EDS were measured under similar excitation conditions: a beam energy of $10 \mathrm{keV}$ and a measuring time of $300 \mathrm{sec}$. A beam current of 10nA was chosen for EDS and $100 \mathrm{nA}$ for WDS, respectively. For WDS two different multilayered crystals were selected and intensities of both were averaged. For EDS an XFlash Silicon Drift Detector with a Mn-K $\alpha$ resolution of $127 \mathrm{eV}$ was used.

In order to evaluate the performance of the WD and ED spectrometers k-ratios are compared and related to the k-ratios given by Bastin [1]. Additionally, quantitative concentrations were determined from WDS and EDS k-ratios and compared to published data of other authors, i.e. Bastin using the same quantification algorithm. Depending on the $\Phi(\rho z)$-methods and mass absorption coefficients (MACs) used considerable deviations are calculated.

The MAC of analyzed transition metals at the B K $\alpha$ energy varies by up to $\pm 20 \%$ which can lead to variations in the resulting concentrations in the same range.

First results of a comparison for chromium boride is presented in Table 1. 
References:

[1] GF Bastin and JM Heijligers in "Quantitative Electron Probe Microanalysis of Boron in Binary Borides", Technische Universiteit Eindhoven (1997) p. 1.

[2] GF Bastin and JM Heijligers, Scanning 12 (1990), p. 225

[3] X Llovet et al., IOP Conf. Series: Materials Science and Engineering 32 (2012), p. 012014.

[4] R Terborg et al., Microsc. Microanal. 22 (S3) (2016), p. 404.

[5] DE Newbury, WM Ritchie, Microsc. Microanal. 21 (2015), p. 1327.

[6] G Castellano et al., Spectrochim Acta B 59 (2003), p. 31.

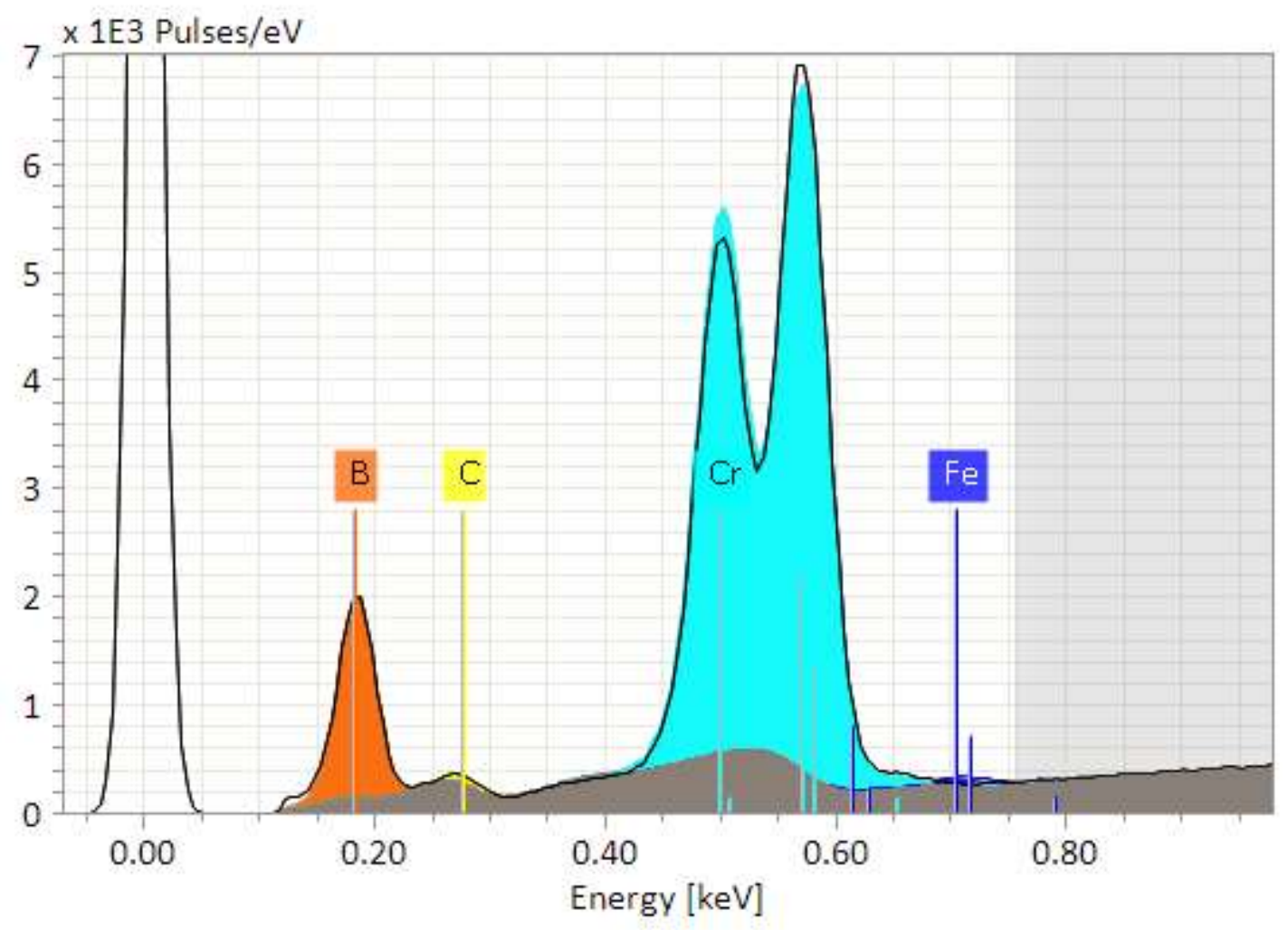

Figure 1. CrB spectrum (black) with calculated Bremsstrahlung background (grey) and peak deconvolution

\begin{tabular}{|c|c|c|c|c|c|c|c|}
\hline CrB & B wt\% & Cr wt\% & Fe wt\% & totals & B at\% & Cr at\% & Fe at\% \\
\hline WDS & 17.7 & 83.3 & 0.1 & 101.1 & 50.6 & 49.4 & 0.1 \\
\hline EDS & 17.7 & 83.4 & 0.2 & 101.3 & 50.5 & 49.4 & 0.1 \\
\hline
\end{tabular}

Table 1. Quantitative analysis of chromium-boride CrB (given concentrations: B: 17.2 wt\%, Cr: 82.8 wt $\%)$ 